\title{
Digital Chest Radiography at the University of Chicago: Present Status and Future Plans
}

\author{
Heber MacMahon
}

\begin{abstract}
Storage-phosphor computed radiography and film digitization systems have been in routine clinical use at the University of Chicago for several years. During this time we have implemented numerous modifications including techniques for scatter reduction, image processing enhancements and display systems to improve the image quality and utility of these devices. We have also evaluated the image quality and functionality of digital systems relative to conventional screenfilm radiography. In this paper, we review our experience and summarize our impressions. In addition, we summarize our plans for a rapid transition into picture archiving and communication systems, with hardcopy interpretation being phased out for most modalities over the next 2 to 5 years.
\end{abstract}

Copyright $₫ 1995$ by W.B. Saunders Company

D IGITAL RADIOGRAPHY systems have been in clinical use at the University of Chicago for approximately 5 years. During this time, we have used both storage-phosphor computed radiography and film digitization, predominantly for bedside radiography. In addition, we have had limited experience using a prototype digital workstation for primary interpretation. The following is a summary of our experience, including the numerous modifications and developments that we have implemented with these systems to enhance their utility. Our plans for implementation of a picture archiving and communication system (PACS) will also be described.

\section{STORAGE-PHOSPHOR COMPUTER RADIOGRAPHY}

We have used a Toshiba 3030A computed radiography (CR) system (Toshiba, Tokyo, Japan) both for general emergency room work and, more recently, for intensive care unit bedside radiography applications. Several modifications have been implemented to improve the clinical utility of the system. In terms of image acquisition, all bedside chest radiography had been performed previously using OC/Lanex Medium film/screen systems (Eastman Kodak, Rochester, NY) with a conventional linear strip antiscatter grid at $90 \mathrm{kV}$. The same exposure conditions, including the grid, have been used for the computed radiography system. Although some degree of interference can be detected on close inspection, grid artifacts caused by interference between the sampling frequency and the grid lines have not been a serious problem. Isodose comparisons of phantom images obtained with and without a grid show a marked improvement for the grid images $(8: 1$ or $10: 1$ focused linear strip grid), particularly in underpenetrated areas of the radiograph.

An early and important modification was establishment of a linkage to our radiology information system (RIS) so that demographic data could be downloaded from the RIS to the CR system upon entry of a three-digit examination code. This has improved the ease and speed of use for technologists.

Like most Fuji based CR systems, the 3030A provided a dual-image hard-copy format with mild and heavily processed versions of the same image at $50 \%$ size reduction. It was our impression that this format was not diagnostically optimal, and there was also a desire to use a single image format for practical reasons. Extensive empirical tests were performed using multiple normal and abnormal images to investigate alternative forms of image processing. Our principal conclusion was that the standard default processing curve, which produced maximum unsharp mask processing in areas with greater exposure, such as the lungs, was undesirable for chest radiography. We have used a highly modified processing curve that applies a $100 \%$ weighting factor from pixel values of 0 to 256 followed by a sharp decrease to a $40 \%$ weighting factor from pixel value 275 to the maximum of 1,023 . In practice, this produces maximum unsharp mask processing in underpenetrated areas, such as the mediastinum and upper abdomen, with very mild processing throughout the lungs. An

From the Department of Radiology, University of Chicago, Chicago, IL.

Address reprint requests to Heber MacMahon, MD, Department of Radiology, University of Chicago, 5841 S Maryland Ave, Chicago, IL 60637.

Copyright (1) 1995 by W.B. Saunders Company

0897-1889/95/0801-1004\$3.00/0 
overall weighting factor of 2.5 is used. A contrast gradient of 0.9 is used, and the mask (kernel) size is not altered.

We performed an observer test to compare this new single-image processing algorithm to the conventional dual-image format. Results for pulmonary nodules, infiltrates, pneumothorax, and location of mediastinal catheters were evaluated. In no category was there a statistically significant difference in detection accuracy. As a result, we have chosen to use the single-image format routinely for several years. We print identical images side-by-side, divide the film with a film cutter, and send the duplicate image to the intensive care unit. We intend to use this form of nonlinear processing for the foreseeable future because we feel it optimizes the information content of chest radiographs without producing disturbing artifacts.

The most recent modification that has been made to our system consists of placement of lead tape material in the back of the CR cassettes to reduce backscatter. It had become apparent that backscatter was a major source of image degradation. Based on a series of phantom experiments, we showed that the backscatter occurred principally because of uncollimated primary radiation hitting the bedframe and being reflected back towards the patient at an oblique angle. The amount of backscatter was sufficient to image metallic components in the back of the cassette in many cases. We found that this could be almost eliminated by careful collimation. However, because collimation is often imperfect in clinical practice, we inserted lead tape inside the backs of the cassettes and this has essentially eliminated the problem. Lead foil is routinely incorporated into the newer $\mathrm{CR}$ cassettes by the manufacturer.

In general, we have found storage-phosphor radiography to provide consistent image quality for bedside radiography, with resulting reduced repeat rates, as well as the ability to produce identical duplicates at low cost.

\section{FILM DIGITIZATION}

We have used film digitization extensively at the University of Chicago for several years. The practical utility of the system has been largely due to the specific features that have been developed in house, including automated density correction and nonlinear digital unsharp mask processing. ${ }^{2,3}$ We have used film digitization in parallel with storage-phosphor computed radiography for bedside radiography applications principally in the intensive care units. The system that we have assembled consists of a Konica laser scanner, and a Sun 3/470 computer linked to a Konica laser printer. As with the CR system, we have used hard-copy output almost exclusively. The acquisition system has consisted of $\mathrm{OC}$ film with Lanex medium screens, focused 8:1 or 10:1 linear strip grids, $90 \mathrm{Kv}$ with variable $\mathrm{mAs}$, and a focus-film distance of $\sim 48$ in. The automated densitycorrection technique, which has been described in detail previously, and the nonlinear unsharp mask processing have produced image quality very similar to that of our CR system. Recently we have implemented a contrast-correction scheme that automatically boosts the image contrast in cases with low subject contrast and/or a misaligned grid. This has contributed to increased image uniformity. The automated density correction has allowed recovery of images with as much as $400 \%$ overexposure and as little as $50 \%$ of normal exposure. As a result, repeat examinations for exposure errors have been virtually eliminated, as with the $\mathrm{CR}$ system.

We print two $50 \%$ reduced hard-copy images from film digitization, one of which is delivered to the Intensive Care Unit (ICU) and one to the Radiology Department. The density-corrected processed digital images are, on average, considered superior to the original radiographs by our chest radiologists. As a result, the digital hard copies are used for primary interpretation and the original films are filed immediately.

Based on our experience, we feel that film digitization is a cost-efficient system. It has a significant advantage over CR in that the original film is available for interpretation when the system malfunctions. The principal disadvantage is that a completely automated commercial system has not been developed. As a result, it is a two-stage process that is more time consuming than CR for the technologist. As we move towards primary interpretation on digital workstations, the fixed film costs and chemical processing associated with film digitization will be significant disadvantages. But while we con- 
tinue to use hard copy for primary interpretation, we consider that film digitization is a practical transition technology.

Our prototype digital workstation, which consists of six 1,000-line monitors in a three-overthree configuration, was built in collaboration with Laboratory Automation Incorporated. Its unique features include its high speed and "friendly" user interface features. It incorporates virtually instantaneous zoom to full $2,000-x$ 2,500-pixel image resolution. Look-up tables for gray-scale adjustment allow rapid alteration of image density. Zoom and gray-scale operations are performed simultaneously on all monitors so that, in the case of a chest radiograph, zooming on one area produces a simultaneous zoom to the same area on the previous comparison radiographs that are displayed alongside. Cases are presented to the radiologist sequentially, as with a conventional film alternator. All functions are mouse or foot-pedal driven. Based on our experience with interpreting ICU chest radiographs, we have formed the impression that this display system is diagnostically comparable with hard copy for bedside chest radiography. We plan more extensive comparisons of soft and hard copy displays in the future.

Because the practicality and cost of a PACS is related to the large amounts of data that need to be transmitted and stored, we have investigated the effects of data compression schemes on image quality. ${ }^{4,5}$ Recently we evaluated an extended Joint Photographic Experts Group compression scheme to examine the relationship between pixel size, compression ratio, and image quality. We performed an observer test using ten radiologists who reviewed 15 images containing subtle nodules, interstitial infiltrates, and pneumothorax. Images were ranked according to perceived quality. One of the most striking conclusions was that $4,000-\times 4,000$-pixel matrix digital chest radiographs that had been compressed up to 30:1 were consistently ranked as equal or superior to uncompressed $2,000-x$ 2,000 -pixel images. When the 4,000- $\times 4,000$ pixel images were reduced to $2,000-\times 2,000$ pixels by pixel averaging, and then compressed as a 2,000- $\times 2,000$-pixel image, compression ratios of greater than 100 to 1 of the original data were achieved with preservation of highly acceptable image quality. In a hospital PACS, such compression would only be performed after interpretation of the original uncompressed image. The compressed images would only be used for comparison purposes so that the minimal loss of image quality would be very unlikely to be clinically significant.

Our immediate future plans include building a PACS which will encompass our existing in-patient facility and a large outpatient facility to be completed in approximately 2 years. Our minimum goal is to distribute all images throughout the outpatient facility over a fiber-optic network for workstation display in the clinics. We anticipate that primary interpretation of cross sectional images such as computed tomography, magnetic resonance imaging, and ultrasound, as well as nuclear medicine, will be possible on digital workstations within 2 years, although it remains to be seen whether soft displays will be accepted for primary interpretation of plain radiographs within this time frame. We expect that workstation interpretation will be gradually introduced into our Radiology Department over the next 5 years. Because all images will be entered into a digital archive, they will be available for immediate review on soft displays, which we anticipate will be used for comparison purposes even for general radiography. This will greatly facilitate the interpretation process and minimize film handling. Although retrieval of previous hard copy from the film file should not be necessary, hard-copy interpretation of the current image may be performed at the radiologist's discretion.

One of our incentives for implementation of workstation interpretation will be the opportunity to implement the numerous computeraided diagnostic schemes that we have been developing for many years. In chest radiology, these include nodule detection, interstitial disease detection and classification, pneumothorax detection, and cardiac size evaluation. ${ }^{6}$ Temporal subtraction, using a warping and automated registration technique to subtract previous from current chest radiographs, will also be implemented for clinical evaluation at the earliest opportunity. ${ }^{7}$ It is our hope that these intelligent workstation features will serve as a catalyst that will accelerate the transition from film to workstation interpretation. 


\section{REFERENCES}

1. MacMahon H, Doi K, Sanada S, et al: Optimal image processing for digital chest radiographs. Image Technol Infor Display. 23:438, 1991

2. Yoshimura H, Xu XW, Doi K, et al: Development of a high-quality film duplication system with a laser digitizer: Comparison with computed radiography. Med Phys 20:5158,1993

3. Hoffmann KR, MacMahon $\mathrm{H}, \mathrm{Xu} \mathrm{XW}$, et al: Evaluation of an enhanced digital film-duplication system with ROC analysis. Invest Radiol 28:1134-1138, 1993

4. MacMahon H, Doi K, Sanada S, et al: Effect of data compression on diagnostic accuracy in digital chest radiogra- phy: Receiver-operating characteristic study. Radiology 178 : 175-179, 1991

5. MacMahon H, Giger ML, Sullivan B, et al: Diagnostic chest radiographs at high-resolution digital sampling. Meeting of the Radiological Society of North America, Chicago, IL, November 27-December 3, 1993

6. MacMahon H, Doi K, Chan HP, et al: Computer-aided diagnosis in chest radiology. $J$ Thor Imaging 5:67-76, 1990

7. MacMahon H, Kano A, Xu XW, et al: Use of difference images for improved detection of interval change in digital chest radiographs. Meeting of the Radiological Society of North America, Chicago, IL, November 27December 3, 1993 